\title{
SHEAR BOND STRENGTH OF METALLIC BRACKETS PHOTO-ACTIVATED WITH LIGHT-EMITTING DIODE (LED) AT DIFFERENT EXPOSURE TIMES
}

\author{
Emanuel Braga RÊGO' ${ }^{1}$, Fábio Lourenço ROMANO ${ }^{2}$
}

\author{
1- Specialist in Orthodontics, School of Dentistry of the Federal University of Alfenas - UNIFAL, Brazil. \\ 2- Master and Doctorate Student of Orthodontics at the Piracicaba Dental School of the State University of Campinas - UNICAMP, Brazil; \\ Professor of Orthodontic at the School of Dentistry, Federal University of Alfenas - UNIFAL.
}

Corresponding address: Avenida Engenheiro José Hebert Faleiros, 600 - Casa 78 - Núcleo São Luís - Ribeirão Preto - SP - 14098-780

Phone: 551639956164 - e-mail: flromano@terra.com.br

Received: October 16, 2006 - Modification: January 29, 2007 - Accepted: July 11, 2007

\begin{abstract}
$T_{\text {ho }}$

he purpose of this study was to compare the shear bond strength of orthodontic metallic brackets photo-activated with two different light-curing sources at different exposure times: halogen light (XL 1500, 3M ESPE) and LED light (Ortholux, 3M Unitek). Sixty bovine permanent lower incisors were inserted into PVC tubes containing plaster. The buccal surfaces were cleaned with pumice and water, and then etched with $37 \%$ phosphoric acid gel. The XT Primer bonding agent (3M Unitek) was applied to the enamel surfaces and the metallic pre-coated brackets (Transbond APC II system, 3M Unitek) were attached to upper central incisors. The teeth were randomly divided into four groups ( $\mathrm{n}=15)$. In Group I (Control), halogen light was used for 40 seconds, while in Groups II, III, and IV were light-cured with LED light unit for 40, 10, and 5 seconds, respectively. The teeth were stored in distilled water at $37^{\circ} \mathrm{C}$ for 24 hours. The brackets were submitted to shear bond strength test in universal testing machine (Instron) at a crosshead speed of $0.5 \mathrm{~mm} /$ minute. Shear bond strength means (MPa) were 4.87 for Group I; 5.89 for Group II; 4.83 for Group III, and 4.39 for Group IV. Tukey's test detected no statistically significant differences among the groups regarding the shear bond strength $(\mathrm{p}>0.05)$. Neither of the types of light-curing sources or exposure times influenced the shear bond strength of metallic brackets.
\end{abstract}

Uniterms: Visible light; Orthodontic brackets; Shear strength.

\section{INTRODUCTION}

The direct bonding of brackets to enamel can be considered an advance for orthodontics because it makes the clinical practice easier and more acceptable among the patients. Since Newman ${ }^{14}$ introduced the direct bonding of orthodontic brackets, different kinds of materials have been proposed for this use, mainly composites photo-activated by halogen light ${ }^{7}$.

Halogen light-curing units have been used in orthodontics for many years and their important characteristics, such as wide-spectrum action, allow any resin material to be polymerized according to different shapes, thus making bracket debonding and damage more difficult. Also, the halogen light-curing units are easy to use and have a low cost maintenance, in addition to being widely employed as controls in several scientific work ${ }^{6}$.

Dunn and Bush $^{8}$ have investigated the use of lightemitting diodes (LEDs) and halogen lights in the polymerization of two composite resins and they found that the halogen lamp was more efficient. Because of the different results found in the literature, the authors emphasized that further studies should be carried out in order to establish the LED effectiveness in replacing conventional halogen light devices.

The halogen bulb generates light as the electrical energy heats a small tungsten filament to high temperatures ${ }^{7}$. The principle of halogen light conversion is not enough because the light power output is $1 \%$ of the total electrical energy consumed ${ }^{1,9}$. The halogen bulb has also a short lifetime (approximated 100 hours) ${ }^{9,12}$ and the output may also be reduced by degradation, reflection, cracked filters, design variation, breakage of optical fibers and tip ${ }^{3}$ and emit a wavelengths of the $410-500 \mathrm{~nm}^{9}$. These features reduce the effectiveness of the halogen light unit in curing dental materials on bovine teeth ${ }^{12}$.

Recently, other curing methods, such as argon laser, xenon plasma-arc, and light-emitting diodes units have been suggested as alternative light sources ${ }^{7,12}$. LEDs are junctions of doped semiconductors that generate light when submitted 
to a low voltage $\mathrm{e}^{10,11}$. The use of LEDs for polymerization of light-activated dental materials was first proposed by Mills ${ }^{13}$. According to the author, new light source based on the use of light-emitting diodes are inexpensive, has long lifetime $\left(10,000\right.$ hours) with little degradation in light output ${ }^{8}$, works with low voltage, and can be designed to emit specific wavelengths (430-480nm), in addition to being compact, and resistant to shock and vibration. The high temperatures generated by the high-power LEDs (900 to $1000 \mathrm{mw} / \mathrm{cm}^{2}$ ) may damage the device, thus requiring maintenance. On the other hand, such a high potency results in faster photoactivation and decreased monomer conversion, which decreases the contraction tension and increases the adhesiveness between brackets and teeth ${ }^{2,10,15,18}$.

Since the LED technology was introduced, studies have been carried out in order to investigate the LED effect on bond strength of orthodontic brackets. Dunn and Taloumis ${ }^{7}$, while evaluating two halogen light-curing units (Optilux 501 and Prolite) and two LEDs (LumaCure and Versalux) with different light intensity, have found no statistically significant difference between them at 40 -second exposure time. Bishara, et al. ${ }^{5}$ compared the same curing devices, both types for 20 seconds and also found no significant difference. Üsümez, et al. ${ }^{19}$ compared halogen light and LED units at 10,20 and 40 seconds of curing time and found that only with 10 seconds of exposure the LED light source showed lower values of shear bond strength. Layman and Koyama $^{10}$, in a clinical setting, concluded that the LED curing unit produced bond strength as strong as those produced by a conventional halogen light, in addition to being faster and more convenient. Cacciafesta, et al. ${ }^{6}$ compared the photo-activation provided by halogen light, LED and plasma-arc units as well as the effect of the light-tip distance on the shear bond strength of orthodontic brackets. These authors found that the mean values of the shear bond strength regarding these three light sources showed no statistically significant difference at $0 \mathrm{~mm}$ distance, but the LED light source had significantly lower mean bond strength values at increased light-tip distances.

The viability of these light sources in bonding brackets has been tested, but few studies have been proposed to investigate the effect of short-term exposure on bracket bonding ${ }^{4,7}$. Therefore, the purpose of this study was to evaluate the shear bond strength of metallic pre-coated brackets photo-activated with LED at different exposure times.

\section{MATERIALAND METHODS}

Sixty freshly extracted bovine permanent lower incisors were collected, cleaned, stored in a $10 \%$ formaldehyde solution for 1 week and kept refrigerated at $6^{\circ} \mathrm{C}$. Only teeth with intact buccal enamel, i.e., no cracks, fissures, or decalcification, were selected for the study. The teeth were randomly divided into four different groups of 15 teeth each. Next, the teeth were inserted into plastic tubes containing plaster (Durone, Dentsply, Petrópolis, Brazil) so that the buccal face of the tooth was perpendicular to the bottom of the tube. All specimens had their buccal enamel surfaces cleaned for 10 seconds with water and fluoride-free pumice (S. S. White, Petrópolis, RJ, Brazil) using a rubber cup and then washed and dried for the same time. In order to standardize the prophylaxis, the rubber cup was replaced every five procedures. The teeth were etched with a $35 \%$ phosphoric acid gel (3M Unitek, Monrovia, CA, USA) for 30 seconds, then washed and dried for 20 seconds. The XT Primer (3M Unitek, Monrovia, CA, USA - bonding agent to be used before bonding the brackets with Transbond XT or Transbond APC II) was applied to the etched enamel using a brush and the material was spread with a mild air stream. The metallic pre-coated brackets for upper central incisors (brackets with Transbond APC II composite attached to the base - Gemini MBT, 3M Unitek, Monrovia, CA, USA) were positioned and the resin in excess removed. Next, they were light cured as follows: In Group I (Control), the specimens were photo-activated with a commercial halogen light (XL 1500, 3M ESPE, Monrovia, CA., USA) for 40 seconds (10 seconds for mesial, distal, incisal and gingival surfaces) at an intensity of $600 \mathrm{~mW} / \mathrm{cm}^{2}$ (according to the manufacturer's instructions). In Groups II, III, and IV, the specimens were light cured with a second-generation LED unit (Ortholux, 3M Unitek, Monrovia, CA., USA) at an intensity of 1000 $\mathrm{mW} / \mathrm{cm}^{2}$ (according to the manufacturer's instructions). The Group II specimens were photo-activated for 40 seconds (10 seconds for each face: mesial, distal, incisal and gingival), whereas the Group III specimens were photo activated for 10 seconds ( 5 seconds for each face: mesial and distal). In Group IV, the specimens were photo activated for 5 seconds ( 3 seconds for mesial face and 2 seconds for distal face). All the light curing procedures were performed as near as possible to the brackets without touching them. After the bonding procedures, the specimens were stored at distilled water and kept in stove at $37^{\circ} \mathrm{C}$ for $24 \mathrm{~h}$, thus simulating the oral conditions. Next, the brackets were submitted to shear strength tests using a universal testing machine (Instron Corp., Canton, Mass.) at a crosshead speed of $0.5 \mathrm{~mm} /$ minute, and the debonding force was applied to the chiselshaped rod put on the bracket. The results were obtained in $\mathrm{kgf}$ and then transformed into MPa for statistical analysis. ANOVA and Tukey's test ( $\alpha=5 \%$ ) were used to detect any significant differences in the shear bond strength among the groups.

\section{RESULTS}

Table 1 presents the shear bond strength means and standard deviation for the four groups. Group II had the highest mean value (5.89 MPa), whereas Group IV had the lowest mean value (4.39 $\mathrm{MPa})$. In spite of this numeric difference, there was no statistically significant differences among the groups $(\mathrm{p}>0.05)$. 


\section{DISCUSSION}

The present study showed no statistically significant differences between the groups, thus demonstrating that exposure time (40, 10 and $5 \mathrm{~s}$.) and type of light-curing device (halogen light or LED) had no influence on the shear bond strength of orthodontic brackets bonded to bovine enamel. These findings were in accordance with studies by Dunn and Taloumis ${ }^{7}$, who used a 40-second exposure time for four different light sources (two LED units and two halogen light-curing units), and Bishara, et al. ${ }^{4}$, who evaluated two devices (one LED unit e one halogen light unit) using a 20second exposure time and found no statistically significant difference. However, such results are also corroborated by Dunn and Busch ${ }^{8}$.

According to Reynolds ${ }^{16}$, a given material can be indicated for clinical use if its bond strength values are around 5.0 $\mathrm{MPa}$ in in vitro investigations. In the present work, only those specimens exposed to LED unit for 40 seconds (Group II) achieved such a value. Although the other groups had lower bond strength values, they were not significantly different and consequently cannot characterize inadequate adhesiveness. The bond strength values found in the present study can be considered low in comparison to other works $\mathrm{s}^{6,7}$ using conventional brackets bonded with Transbond XT instead of Transbond APC. Although Oesterle, et al. ${ }^{15}$ had used brackets similar to those employed in the present study, the bond strength values were also found to be lower, mainly due to the different manufacturers. On the other hand, the absolute values found in the present study are similar to those observed by Bishara, et al. ${ }^{4}$, who used APC bracket system.

The bond strength values obtained at 5 and 10 seconds with Ortholux LED, which is a high-power device (1000 mw/ $\mathrm{cm}^{2}$ ), can be explained by the high density, a condition that favors the rapid initial monomer polymerization. As a result, monomer consumption is impeded by their movement and polymerization would be almost immediate. On the other hand, lower density favors greater monomer mobility, thus forming a greater number of polymeric chains and increased polymerization contraction ${ }^{15}$. This irradiance value is much greater than the one usually indicated as appropriate for light curing dental composites, approximately $300 \mathrm{mw} / \mathrm{cm}^{2}$, as described by Barghi, et al. ${ }^{3}$ As the high-power LED unit

TABLE 1- Descriptive statistics and the results of Tukey's test comparing the shear bond strengths $(\mathrm{MPa})$ of the tested groups

\begin{tabular}{lcc} 
Group & Mean (Standard deviation) & Test* $^{*}$ \\
\hline I & $4.87(1.98)$ & $\mathrm{a}$ \\
II & $5.89(1.93)$ & $\mathrm{a}$ \\
III & $4.83(1.81)$ & $\mathrm{a}$ \\
N & $4.39(1.22)$ & $\mathrm{a}$ \\
\hline
\end{tabular}

${ }^{* *}$ All values were statistically equivalent $(p>0.05)$ generates much heat, it requires constant refrigeration in order to avoid chip degradation and consequent equipment repair. Dunn and Taloumis ${ }^{7}$ compared a $150 \mathrm{~mW} / \mathrm{cm}^{2}$ LED unit to two halogen light-curing units, one with $1030 \mathrm{~mW} /$ $\mathrm{cm}^{2}$ and other with $400 \mathrm{~mW} / \mathrm{cm}^{2}$. They found no statically significant differences in the bond strength values, thus raising the question of whether high potency is really necessary for light curing the material to bond orthodontic brackets.

Although some pen-shaped LED units have been launched to the market, these recent devices have been yielding results similar to those obtained with halogen lightcuring units if one considers the frequent fractures and debonding regarding the former. Despite the divergent opinions, one of the few advantages of the LED units in comparison to halogen light units ${ }^{8,15}$ is the lifetime of the diodes and the possibility of reducing the photo-activation time, which was observed in the present work as well.

The results of the present study also demonstrated that the bond strength was not significantly influenced by the reduction of the curing time. Layman and Koyama ${ }^{10}$, in a clinical setting, compared the 40-second cure time using halogen light to 10 -second curing time using a LED device. Bonding failures recorded over a three-month period were submitted to statistical analysis, and the LED device showed a better performance, despite the lack of statically significant differences.

On the other hand, the results of the present study are not consistent with those by Swanson, et al. ${ }^{17}$, who found higher bond strength values when the curing time was extended. According to the authors, the composite layer between the bracket and tooth is sufficiently thin to allow that an adequate monomer conversion occur even using commercial LED devices. Also, the low mean values were found to be acceptable for orthodontic use. Üsümez, et al. ${ }^{19}$ compared halogen light devices to LED units at 10, 20, and 40 seconds of curing time and found that only the LED at 10-second exposure time showed lower shear bond strength values, which is not corroborated by the present study, as the shear bond strength values for shorter exposure times ( 5 and 10 seconds) were similar to those obtained at 40 seconds. This statistical equivalence among the experimental results obtained with different exposure times was probably due to the proximity between light source and bracket as well as to the small thickness of the bonding material used for attachment to tooth surface.

\section{CONCLUSIONS}

Exposure time had no influence on the bond strength values of brackets bonded with photo-activated composite using LED source, as the results were similar to those obtained with halogen light at 40 seconds. Bonding precoated brackets with LED device within shorter exposure periods ( 5 or 10 seconds) seems to be a good alternative for reducing chairtime in the daily orthodontic practice, but further studies are still necessary if such a technique is to 
be recommended. In summary, despite the emergence of novel light source units on the market, the halogen light still provides good results when compared to the new lightcuring devices.

\section{REFERENCES}

1- Althoff O, Hartung M. Advances in light curing. Am J Dent. 2000;13(sp. issue):77-81

2- Bang HC, Lim BS, Yoon TH, Lee YK, Kim CW. Effect of plasma arc curing on polymerization shrinkage of orthodontic adhesive resins. J Oral Rehabil. 2004;31:803-10.

3- Barghi N, Berry T, Hatton C. Evaluating intensity output of curing lights in private dental offices. J Am Dent Assoc. 1994;125:9926.

4- Bishara SE, Ajlouni R, Oonsombat C. Evaluation of a new curing light on the shear bond strength of orthodontic brackets. Angle Orthod. 2003;73:431-5.

5- Bishara SE, Olsen M, Von Wald L. Comparisons of shear bond strength of precoated and uncoated brackets. Am J Orthod Dentofacial Orthop. 1997;112:617-21

6- Cacciafesta V, Sfondrini MF, Scribante A, Boehme A, JostBrinkmann PG. Effect of light-tip distance on the shear bond strengths of composite resin. Angle Orthod. 2005;75:352-7.

7- Dunn WJ, Taloumis LJ. Polymerization of orthodontic resin cement with light-emitting diode curing units. Am J Orthod Dentofacial Orthop. 2002;122:236-41.

8- Dunn WJ, Bush AC. Comparison of polymerization by lightemitting diode and halogen-based light curing units. J Am Dent Assoc. $2002 ; 133: 335-41$

9- Fujibayashi K, Ishimaru K, Takahashi N, Kohno A. Newly developed curing unit using blue light-emitting diodes. Dent Jap. 1998;34:4953 .

10 - Layman W, Koyama T. A clinical comparison of LED and halogen curing units. J Clin Orthod. 2004;37:385-7.

11 - Malvino AP. Eletronic. São Paulo: Makron books; 1995.

12 - Mills RW, Jandt KD, Ashworth SH. Dental composite depth of cure with halogen and blue light emitting diode technology. Br Dent J. 1999;186:388-91.

13- Mills RW. Blue light emitting diode- an alternative method of light curing [letter]? Br Dent J. 1995;178:169.

14- Newman GV. Bonding plastic orthodontic attachments to tooth enamel. J New Jersey Dent Soc. 1964;35:346-458.

15 - Oesterle LJ, Newman SM, Shellhart WC. Rapid curing of bonding composite with a xenon plasma arc light. Am J Orthod Dentofacial Orthop. 2001;119:610-6.

16- Reynolds IR. A review of direct orthodontic bonding. Br J Orthod. $1975 ; 2: 171-8$

17- Swanson T, Dunn WJ, Childers DE, Taloumis LJ. Shear bond strength of orthodontic brackets bonded with light-emitting diode curing units at various polymerization times. Am J Orthod Dentofacial Orthop. 2004;125:337-41.
18- Tarle Z, Meniga A, Knezevic A, Sutalo J, Ristic M, Pichler G. Composite conversion and temperature rise using a conventional plasma arc and experimental blue LED curing unit. J Oral Rehabil 2002;29:662-7.

19- Usumez S, Buyukyilmaz T, Karaman AL. Effect of light-emitting diode on bond strength of orthodontic brackets. Angle Orthod. 2004;74:259-63. 